Société d'histoire de la révolution de 1848 et des

révolutions du XIXe siècle

$58 \mid 2019$

Histoire et archéologie : que faire du XIXe siècle ?

\title{
"Traités comme des pigeons ». Pratiques commerciales et marché estudiantin à Oxford à l'époque victorienne
}

Business Practices and the Student Market in Nineteenth-Century Oxford Geschäftspraktiken und Studentenmarkt in Oxford während der Viktorianischen Ära

\section{Sabine Chaouche}

\section{OpenEdition}

Journals

Édition électronique

URL : https://journals.openedition.org/rh19/6411

DOI : 10.4000/rh19.6411

ISSN : $1777-5329$

Éditeur

La Société de 1848

Édition imprimée

Date de publication : 1 août 2019

Pagination : 209-227

ISSN : 1265-1354

\section{Référence électronique}

Sabine Chaouche, « «Traités comme des pigeons ». Pratiques commerciales et marché estudiantin à Oxford à l'époque victorienne », Revue d'histoire du XIXe siècle [En ligne], 58 | 2019, mis en ligne le 01 janvier 2022, consulté le 08 juillet 2022. URL : http://journals.openedition.org/rh19/6411 ; DOI : https://doi.org/10.4000/rh19.6411 


\section{SABINE CHAOUCHE}

\section{"Traités comme des pigeons». Pratiques commerciales et marché estudiantin à Oxford à l'époque victorienne}

Les freshers sont "traités comme des pigeons ${ }^{1}$ » : tel était le sentiment d'un jeune diplômé sur la manière dont les collèges se conduisaient à l'égard des nouveaux arrivants venus étudier à Oxford. Dans son article du 22 novembre 1865 paru dans Punch, il suggérait que les étudiants étaient victimes de pratiques éhontées visant uniquement à leur extorquer de l'argent : trop de nourriture était intentionnellement servie dans les halls, le surplus étant donné aux serviteurs après les repas, et par conséquent surfacturé. De fait, les étudiants payaient indirectement le salaire des serviteurs travaillant dans les collèges, ces derniers n'étant pas ou que peu rémunérés. Ce témoignage traduit de façon implicite et brutale l'importance du marché estudiantin à Oxford, notamment son potentiel lucratif, le nombre d'inscrits ayant doublé entre 1800 et $1860^{2}$ et étant pour une très grande majorité d'entre eux issus d'un milieu aisé, et donc virtuellement de bons futurs «clients». Ainsi, la venue régulière de nouveaux étudiants aiguisait les appétits des collèges - mais également des marchands, la consommation estudiantine ayant des répercussions sur l'ensemble des activités de la ville universitaire. Ces adolescents se voyaient allouer en moyenne 200 livres chaque année par leurs parents (500 livres pour les plus riches) pour payer leurs battels, c'est-à-dire leur nourriture et boissons au collège ainsi que leurs frais de scolarité3. Une

1. Punch, 22 novembre 1865 .

2. L'Université d'Oxford comprenait au XIX ${ }^{e}$ siècle une vingtaine de collèges (Keble ayant été construit dans la seconde moitié du siècle) et quelques Halls (New Inn, St Alban, St Edmund dit «Teddy Hall» et St Mary). Le nombre d'étudiants ne cessa de croître au cours du siècle : d'après nos estimations fondées sur les registres d'inscription, on comptait un peu plus de 6000 étudiants dans les années 1800 ; ce chiffre doubla dans les années 1820 et resta relativement stable jusque dans les années 1860 qui furent une période de réformes. Plus d'étudiants furent autorisés à s'inscrire. De fait, la population estudiantine comptait plus de 22000 personnes dans les années 1890 .

3. Les frais de scolarité («matriculation», frais d'inscription de l'Université et du Collège) étaient inférieurs à 20 livres tout au long du siècle. Ces derniers ne comprenaient ni le logement, ni les meubles des chambres, ni la nourriture, ni d'autres dépenses comme les pourboires donnés aux serviteurs, le nettoyage et le repassage du linge et bien entendu les fêtes organisées régulièrement, appelée wines. D'après Laurence Brockliss, la moyenne de l'ensemble des frais occasionnés s'élevait à 150 livres par an par étudiant mais il n'était pas rare qu'ils atteignissent 200 à 300 livres (The University of Oxford: A History, Oxford, Oxford University Press, 2016, p. 271 et sur l'évolution de 
partie de cet argent servait aussi d'argent de poche pour aller faire les boutiques, que cela soit pour l'achat de vêtements, d'accessoires - comme par exemple des cigarettes, du papier à lettre, des objets décoratifs, du parfum -, ou de biens périssables. La présence de nombreux marchands dans la ville (près de 1700 dans les années 1860) ${ }^{4}$ et l'importance du marché estudiantin posent la question des pratiques commerciales spécifiquement développées pour faire de ces jeunes hommes des clients mais également des consommateurs invétérés.

Victoria de Grazia et Ellen Furlough affirment que la consommation est par essence féminine ${ }^{5}$. Cette perspective, dominante dans les études sur le shopping au XIX ${ }^{\mathrm{e}}$ siècle, explique le fait que les historiens de la consommation et du commerce se soient focalisés sur les femmes et leurs emplettes ${ }^{6}$, voire leur passion pour les grands magasins et pour la mode, mais non pas sur les hommes, et encore moins les adolescents ${ }^{7}$. Pourtant ces derniers,

ces coûts, p. 271-274). Les familles les plus aisées pouvaient dépenser de telles sommes, aussi la très grande majorité des étudiants appartenait à l'aristocratie (upper class). La population estudiantine était tripartite, composée également de fils de pasteur (clergymen's sons) et de bourgeois que l'on nommait soit gentlemen commoners (ceux dont les parents avaient un certain statut dans la société ou de hauts revenus), soit commoners (ceux qui appartenaient à la classe moyenne ou middle class).

4. D’après nos estimations basées sur les données des annuaires Pigot et Kelly des professions de la ville :

\begin{tabular}{|c|c|c|}
\hline Année & Nombre de marchands & \% de + ou de - par rapport à la dernière recension \\
\hline 1823 & 780 & 17,9 \\
\hline 1830 & 920 & 55,5 \\
\hline 1847 & 1431 & $-28,5$ \\
\hline 1854 & 1023 & 62,8 \\
\hline 1869 & 1666 & $-16,5$ \\
\hline 1877 & 1391 & 88,9 \\
\hline 1891 & 2628 & \\
\hline
\end{tabular}

Noter que ces estimations sont imparfaites et doivent être relativisées dans la mesure où certains marchands n'étaient pas recensés dans ces annuaires. Par ailleurs, un même marchand pouvait avoir plusieurs boutiques différentes.

5. Victoria De Grazia, et Elen Furlough (dir.), The Sex of Things: Gender and Consumption in Historical Perspective, Berkeley, CA; Londres, University California Press, 1996, p. 1.

6. Voir Dorothy Davis, A History of Shopping, Londres, Routledge \& Kegan Paul Ltd., 1966; Alison Adburgham, Shopping in Style: London from the Restoration to Edwardian Elegance, [London], Thames and Hudson, 1979; Erika D. Rappaport, "'The Halls of Temptation' : Gender, Politics, and the Construction of the Department Store in Late Victorian London ", Journal of British Studies, t. 35, $\mathrm{n}^{\circ}$ 1, 1996, p. 58-83 et Shopping for Pleasure: Women in the Making of London's West End, Princeton, Princeton University Press, 2001; William Lancaster, The Department Stor : A Social History, Leicester, Leicester University Press, 1995; M. E. Baren, Victorian Shopping, Londres, Michael O’Mara, 1998; Alexander Nicholas, The Emergence of Modern Retailing, 1750-1950, Londres, Frank Cass, 1998; Tammy C. Whitlock, Crime, Gender, and Consumer Culture in Nineteenth-Century England, Aldershot, Ashgate, 2005; Kelly Graham, Gone to the Shops : Shopping in Victorian England, Westport, CT, Londres, Praeger Publishers, 2008.

7. Peu d'ouvrages ont abordé le sujet. Voir Christopher Breward, The Hidden Consumer: Masculinity, Fashion and City Life 1860-1914, Manchester, Manchester University Press, 1999, en particulier le chapitre 5 sur les jeunes hommes; Brent Shannon, The Cut of his Coat, Men, Dress and Consumer Culture, in Britain, 1860-1914, Athens, Ohio University Press, 2006 et Laura Ugolini, Men and Meanswear. Sartorial Consumption in Britain, 1880-1936, Farnham, Ashgate, 2007. Les auteurs 
en particulier les étudiants, participaient activement au développement de la ville où ils résidaient temporairement, étant, au même que titre que les adultes ayant des revenus réguliers, des acteurs économiques. Quelles furent dès lors les relations entre marchands et étudiants? Que traduisent-elles en matière économique et en quoi reflètent-elles l'émergence du capitalisme au $\mathrm{XIX}^{\mathrm{e}}$ siècle? Quelles conséquences eurent-elles sur le niveau de consommation des étudiants? Et finalement, furent-ils eux aussi réellement «traités comme des pigeons" par les marchands?

Cet article examine les stratégies mises en place pour pousser des adolescents à la consommation. Il se fonde sur des documents d'archives et sur des articles parus dans les journaux qui témoignent des pratiques commerciales développées par les marchands à l'époque victorienne. Celles-ci visaient d'une part à faire face à une concurrence de plus en plus forte entre marchands d'une même profession, et d'autre part à sécuriser de façon rapide de nouvelles commandes par les étudiants. Si les dépenses excessives et ostentatoires de ceux-ci reflétaient un goût certain pour le gaspillage et la prodigalité comme en témoigne l'exemple d'Edward Napleton Jennings dont les vicissitudes financières furent mises en lumière par la presse ${ }^{8}$, on peut néanmoins affirmer qu'elles étaient également le résultat de méthodes capitalistes agressives, comme la distribution directe et le harcèlement commercial (méthodes appelées touting et dunning). Plusieurs milliers d'étudiants furent ainsi poursuivis en justice à Oxford au XIx ${ }^{\mathrm{e}}$ siècle ${ }^{9}$. En effet, les facilités de paiements offertes par les marchands à une clientèle jeune et inexpérimentée avaient un impact majeur sur la consommation, en stimulant mécaniquement la demande. Le crédit fut souvent à l'origine de dépenses excessives et de l'endettement, voire du surendettement, et ce, bien avant les années 1920. De façon plus générale, son utilisation ne se limitant pas à ce groupe particulier que représentaient les étudiants, il apparaît un levier de ce qui deviendra la consommation de masse. Il met ainsi en lumière la manière dont ont pu se développer des comportements consuméristes et la genèse de la société de consommation, triomphante au $\mathrm{Xx}^{\mathrm{e}}$ siècle.

examinent les stéréotypes de classe auxquels les hommes étaient confrontés, les spécificités de la mode masculine et leur impact sur leur relation aux vêtements, ainsi que le commerce des habits masculins et leur promotion.

8. Par exemple, en 1858, le fils de John Scratton avait une dette de 96648 livres (la plus grande partie contractée à l'Université), dont 49000 livres d'intérêt dus aux usuriers. Voir également Oxford Journal, 11 juillet 1835, Joseph Sladen Smith ayant une dette de $£ 1505$ 6s 8d; Morning Chronicle, 4 septembre 1849, dette de 764 livres; Reynolds's Newspaper, 11 janvier 1852, dette de 1700 livres; Western Times, 26 mai 1855, étudiant de Balliol College qui, au cours de quatre années passées à Oxford dépensa 4000 livres et cumula $£ 1780$ 1s 9d de dettes; Reynolds's Newspaper, 27 juillet 1856, dette de 1400 livres; Liverpool Daily Post, 16 novembre 1866, faisant allusion à un étudiant ayant dépensé 1000 livres par an; Oxford University and City Herald, 16 mars 1867, dette de 3017 livres dont 2871 livres dues à des usuriers etc.

9. Archives de l'Université d'Oxford, Chancellor's Court Papers, 1800-1899. Plus de sept mille dossiers furent traités au cours du siècle par la cour de justice (noter que certains étudiants furent parfois poursuivis par plusieurs marchands). 


\section{Consommation, ÉMULATION ET COMPÉTITION}

En janvier 1848, Edward Nappleton Jennings, alors âgé de 22 ans et fils de pasteur, comparut en justice à Londres, ayant une dette de 1865 livres - dont 1697 livres dues aux marchands d'Oxford - contractée entre 1845 et 1847 , alors qu'il faisait ses études à Worcester College ${ }^{10}$. Il avait bénéficié d'un subside de 250 livres alloué annuellement par son père. La presse donna un compte rendu détaillé de ses dépenses, parmi lesquelles $£ 56$ en vin, $£ 33$ en équipement équestre, $£ 40$ en billards, $£ 46$ en confiseries et pâtisseries, $£ 301$ en vêtements et $£ 98$ en chapeaux. Jennings fut exclu de l'Université et abandonna finalement ses études. Ces achats suggèrent qu'il prit un certain plaisir à dépenser sans compter mais surtout qu'il tenta de s'identifier aux codes de l'élite. Le type d'achats effectués auprès des marchands, principalement liés aux loisirs et à l'agrément laisse entendre qu'il avait adopté un train de vie aristocratique, peu en adéquation avec ses revenus ${ }^{11}$. Il avait aussi, semble-t-il, particulièrement soigné son apparence, les jeunes gens étant très sensibles à la mode comme le souligne Paul Deslandes dans son ouvrage sur les étudiants d'Oxbridge ${ }^{12}$. Il tâcha le plus probablement de se conformer à la pression sociale exercée par la communauté estudiantine en matière de consommation et de style de vie. En effet, l'émulation entre étudiants est comparable à ce que le sociologue et économiste Thorstein Veblen a qualifié à la fin du XIX $\mathrm{X}^{\mathrm{e}}$ siècle de consommation ostentatoire (conspicuous consumption) en évoquant le cas des nouveaux riches aux États-Unis qui exhibaient leur statut et leur grande fortune à travers leurs achats et le gaspillage de leur argent ${ }^{13}$. L'étalage de luxe participe ainsi d'une construction sociale du statut et devient symboliquement une marque de pouvoir. En ce sens, il est l'affirmation de ce que Pierre Bourdieu nomma jadis le «capital économique" d'un individu ${ }^{14}$. Devenir populaire auprès de ses pairs à Oxford signifiait qu'il fallait se conformer à l'habitus masculin mondain, c'est-à-dire à la fois afficher une identité précise, celle du gentleman, et une classe, celle des élites. En effet, la culture estudiantine se voyait dominée par les classes supérieures, les jeunes gens issus de l'aristocratie étant majoritaires dans la première partie du siècle comme le montre l'étude de Lawrence Stone ${ }^{15}$. Ainsi, le fait même de consommer et dans une certaine mesure de gaspiller,

10. Voir le résumé de l'affaire Jennings dans l'article de Mark C. Curthoys et Chris J. Day, "The Oxford of Mr Verdant Green", in M. G. Brock et Mark C. Curthoys (dir.), The History of the University of Oxford, Oxford, Oxford University Press, 2000, vol. 6, p. 277-278.

11. Voir la liste de ses achats en annexe.

12. Paul R. Deslandes, Oxbridge Men, Indiana, Indiana Press, 2007, p. 62-82.

13. Thorstein Veblen, The Theory of the Leisure Class, New York, 1899. 1986.

14. Pierre Bourdieu, Distinction. A Social Critique of the Judgement of Taste, Londres, Routledge,

15. À partir des années 1795 , le nombre de nouveaux étudiants issus de la noblesse passa de 70 par an, à 130 en 1810, et de 130 à 190 à la fin des années 1830 (Lawrence Stone (dir.), The University in Society: Oxford and Cambridge from the 14th to the Early 19th century, Oxford, Oxford University Press, 1999, p. 14 et 93). Cette prédominance de l'aristocratie semble avoir été unique en Europe. 
était-il perçu comme un signe et un marqueur de richesse. L'achat d'objets onéreux s'avérait indispensable pour certains qui souhaitaient se positionner au sein même du groupe. Nombre d'étudiants venaient à Oxford non pas seulement pour y obtenir un diplôme mais également pour se faire des relations, aussi beaucoup devaient faire face aux tentations du monde matériel et aux dangers liés à la prégnance de l'apparence dans le développement des relations sociales. Nombreux furent ceux qui, comme Edward Jennings, désirant mettre en scène une (feinte) opulence, s'accoutumèrent à faire étalage de luxe et donc à s'endetter rapidement comme le montrent les archives de l'Université d'Oxford ${ }^{16}$.

L'insolvabilité de Jennings fit scandale. Son procès fut rapporté au plan national par de nombreux journaux qui soulignèrent les périls auxquels étaient confrontés les mineurs ${ }^{17}$. Les journalistes arguèrent que les marchands séduisaient leurs proies ${ }^{18}$, et que ces jeunes hommes inexpérimentés et sans défense, encouragés à dépenser de façon extravagante prenaient un chemin hasardeux, celui de la perdition morale et financière ${ }^{19}$. "En Route Vers La Ruine» titrait ainsi le Blackburn Standard daté du 5 janvier 1848, suggérant que les marchands poussaient délibérément les adolescents à consommer. Ce point de vue ne varia guère au cours du siècle : les journalistes restèrent très partiaux, se refusant à entendre le point de vue des commerçants ou même à admettre que certains étudiants pussent être prodigues. Quelles furent réellement ces pratiques commerciales et avaient-elles en effet pour but ou pour effet de stimuler la consommation?

Le Blackburn Standard (5 janvier 1848) rapporta les faits suivants concernant la première semaine que passa Jennings à Oxford : «dès qu'il entra dans sa chambre, un certain nombre de marchands" - une cinquantaine apparemment - «lui rendirent visite pour qu'il devienne leur client ${ }^{20}$ ", ajoutant qu'il y avait des marchands de vin, des tailleurs, des bottiers et des vendeurs de cigares. Ils venaient ainsi directement montrer leur marchandise aux fres-

En effet, les jeunes nobles avaient le plus souvent un tuteur et se voyaient éduqués dans la demeure familiale.

16. La Chancellor's Court était une cour de justice spécifique à l'Université d'Oxford qui passait en revue les cas de dettes et qui aidait les créanciers à recouvrer les sommes d'argent qui leur étaient dues. Cette cour avait un rôle important puisqu'elle était essentiellement destinée à contrôler indirectement le marché en aidant les marchands à récupérer leur argent. Les poursuites judiciaires à l'encontre des étudiants augmentèrent considérablement à partir de 1830, suggérant de nouveaux comportements en matière de consommation, notamment des excès. Voir l'article de Chris Day, "The University and the City", in M. G. Brock and M. C. Curthoys (dir.), op. cit., vol. 6, p. 442 et Malcolm Underwood, "The structure and operation of the Oxford Chancellor's Court from the sixteenth to the early eighteenth century", Journal of Society of Archivists, t. 6, n 1, 1978, p. 19-27.

17. Un étudiant devenait majeur à l'âge de 21 ans.

18. Cork Examiner, 12 janvier 1848.

19. Les journaux suivants mentionnent le procès de Jennings : Blackburn Standard, Hertford Mercury and Reformer, London Daily News, Cambridge Chronicle and Journal, Cork Examiner, Lancaster Gazette, Lloyds Weekly Newspaper, Hampshire Advertiser, Dundee, Perth and Cupar Advertiser, London Standard, Morning Post, Oxford Journal, Stamford Mercury, The Era, Yorkshire Gazette.

20. Nous traduisons. Citation originale : «as soon as he entered his apartments, he was visited by a number of tradesmen soliciting custom ". 
hers afin de les compter parmi leurs nouveaux clients. Ce ballet incessant de marchands, allant et venant dans les collèges, signale, d'une part, que ces pratiques étaient tolérées par l'Université, et, d'autre part, que les marchands préféraient le démarchage et la vente directe, pratique commerciale que l'on appelait à l'époque «touting ${ }^{21}$ ». Cette forme de vente, comparable aux techniques du porte à porte des colporteurs semble avoir été cruciale en matière commerciale, les étudiants représentant pour ces négociants une manne financière dont il fallait se saisir rapidement, d'où leur empressement à rencontrer les nouveaux venus. La presse y vit une forme de harcèlement pour faire pression sur des jeunes hommes, pour la plupart ignorant tout des règles du commerce. En effet, d'après le London Daily News ( $1^{\text {er }}$ janvier 1848), Jennings dut faire face à une telle opiniâtreté des marchands qu'il se crut obligé de commander des articles sans en éprouver réellement le besoin. Il s'agissait bien là, selon toute vraisemblance, de ventes forcées. En effet, Jennings inféra que les vendeurs réitéraient leurs visites jusqu'à ce que, exténué, l'étudiant cède à leurs demandes.

Cette forme de harcèlement montre à quel point avoir pour clients ces jeunes hommes était fondamental pour les commerçants. Elle avait déjà été rapportée par un ancien étudiant quatre ans plus tôt dans son ouvrage Tradesmen and Undergraduates ${ }^{22}$. Dès le début du trimestre, chaque fresher recevait une liste des fournitures et vêtements nécessaires pour ses études, de même qu'une liste de marchands recommandés par l'Université (sous licence). En entrant dans sa chambre, il pouvait voir son bureau recouvert de cartes de visite le sollicitant de bien vouloir devenir client. Cependant, une fois les cours commencés, une cohorte de vendeurs se pressait à sa porte, certains d'entre eux laissant même des articles, comme par exemple des encyclopédies ou des vêtements, afin qu'ils soient évalués par le futur consommateur. Cette tactique se révélait en réalité une forme d'escroquerie, l'étudiant malheureux devenant, à son insu, débiteur. En effet, le vendeur se gardait de venir récupérer l'article considérant qu'il n'était pas prêté mais bel et bien commandé. En revanche, il ne manquait jamais de rapporter, en l'absence de l'étudiant, un autre volume de la série ou un autre habit, flouant ainsi de façon répétée son nouveau client ${ }^{23}$. Ces pratiques déshonnêtes signalent à quel point le marché était compétitif à Oxford mais également rentable pour ceux qui savaient "tirer leur épingle du jeu ». Selon toute vraisemblance, le marché estudiantin permit à certains commerçants de s'enrichir de façon conséquente : William Dyes, tailleur tenant boutique sur Beaumont Street, mourut en 1871 en laissant un capital de 120000 livres; la fortune d'Henry Moses Gamon, mar-

21. Voir par exemple le Morning Post du 19 mai 1849.

22. Tradesmen and Undergraduates, or the Present System. Debt and Credit at Oxford Unveiled by an Oxford B.A., Londres, John Ollivier, 1844.

23. Tradesmen and undergraduates, op. cit., p. 4. 
chand de vin sur Cornmarket Street, fut quant à elle estimée à 40000 livres après son décès ${ }^{24}$.

L'aspect cyclique de l'économie d'une ville universitaire comme Oxford contribuait à renforcer ces pratiques commerciales agressives, dans la mesure où la consommation était stimulée de façon régulière, presque mécaniquement pourrait-on avancer. Certains étudiants quittaient la ville, au moment où d'autres venaient s'y installer. Il était donc crucial d'accaparer de nouvelles parts de marché au bon moment. Par ailleurs, des changements d'ordre juridique s'étaient produits une dizaine d'années auparavant, ayant un impact majeur sur le commerce à Oxford. En 1835 le Municipal Corporations Act abrogea le droit d'exclusivité dont bénéficiaient certains marchands d'Oxford, décision qui créa un appel d'air : des commerçants se saisirent de l'occasion et vinrent y ouvrir boutique. Cet afflux massif de nouveaux marchands renforça de façon sensible la concurrence. Entre 1830 et 1847, le nombre de magasins passa de 920 à $1431^{25}$, augmentant donc de près de $56 \%$, ce qui explique l'acharnement des vendeurs et leur volonté de privilégier, dans la ville même, le démarchage en apportant directement les biens de consommation dans les chambres des étudiants. Enfin, le développement du chemin de fer, ouvert le 12 juin 1844 à Oxford, de même que le solide réseau de transport reliant la capitale aux provinces britanniques, joua également un rôle important en matière de concurrence. Certains négociants se rendaient spécialement à Oxford au début de chaque trimestre afin d'y faire des affaires, déstabilisant le marché. D'après l'auteur de Tradesmen and Undergraduates, leurs produits étaient de très médiocre qualité mais les prix quant à eux s'avéraient exorbitants ${ }^{26}$. Il semble, une fois de plus, que les marchands cherchaient à tirer parti de l'inexpérience des étudiants et de leur manque de connaissances en matière de consommation, notamment en ce qui concerne la valeur et les prix des produits en circulation, pour faire d'importants profits.

Ces pratiques commerciales agressives pourraient être comparées aux stratégies push-pull utilisées de nos jours en matière de marketing. En effet, de nombreux marchands ne manquaient pas de promouvoir leurs produits en allant distribuer leurs catalogues aux étudiants et en leur vantant la qualité de leurs produits, mais aussi en donnant leur carte de visite aux collèges ou en passant des annonces dans les journaux estudiantins comme The Undergraduatés Journal par exemple, de façon à attirer les consommateurs dans leurs magasins. Ils pouvaient ainsi stimuler doublement la demande. En outre, si le fait de se déplacer chez un riche client était une pratique courante comme le montre Helen Berry, il apparaitt que, dans le cas du commerce à Oxford

24. Cheltenham Looker, 26 août 1871.

25. Ces chiffres sont tirés de l'annuaire Pigot (J. Pigot, Directory of Oxfordshire 1830, Cinderford, Archive CD Books et Directory of Oxfordshire 1842/44, Cinderford, Archive CD Books). Les tavernes ne sont pas incluses.

26. Tradesmen and undergraduates, op. cit., p. 5-6. 
et à la différence des traditions commerciales avec l'aristocratie, la grande majorité des freshers ne sollicitait pas les marchand ${ }^{27}$. Ils n'éprouvaient donc pas un besoin impérieux de commander des articles depuis leur chambre, préférant sans doute aller flâner dans les rues pour découvrir la ville et ses boutiques. Les marchands, stratégiquement, cherchaient à élargir leur clientèle en misant sur les achats impulsifs, voire compulsifs, peu d'étudiants étant versés dans l'art du négoce et des négociations. Selon Laermans, il était ainsi d'usage d'estimer la valeur des produits et de discuter du prix à payer ${ }^{28}$. La plupart des freshers n'étaient probablement guère familiers de ces usages (sauf à avoir accompagné leurs parents ou assisté à des ventes par le passé) et se gardaient de marchander, voire même de demander le prix des produits qu'on leur proposait d'acheter. Certes, d'anciens étudiants pouvaient mettre en garde les nouveaux venus ou les aider à résister à la tentation comme il apparaît par exemple dans le roman Tom Brown at Oxford (1861) de Thomas Hughes $^{29}$. Cependant, il fallait un certain temps pour que les cercles d'amis se forment, les freshers étant progressivement invités à des soirées tout au long du trimestre. Ainsi, les toutes premières semaines s'avéraient cruciales en matière de ventes.

Face à ces témoignages, il apparaît que certaines pratiques commerciales exercées dans les collèges relevaient d'une concurrence exacerbée entre marchands résidant à Oxford ou provenant de différentes villes et avaient pour origine une libéralisation d'un marché dominé par une politique du «laissezfaire» et donc par un manque de régulations et de réglementations. L'affaire Jennings qui n'était pourtant pas un cas isolé, parce qu'elle eut un impact national, amena précisément les marchands d'Oxford à revoir leurs pratiques commerciales tout en défendant leur intégrité.

\section{Mesures de protection et tentatives POUR RÉGUler LE MARCHÉ ESTUDIANTIN}

Thomas Randall (1805-1887), futur maire d'Oxford, fut accusé d'extorsion, son magasin étant d'ailleurs surnommé par les étudiants «La boutique de la ruine» (the ruination shop). De fait, il publia une réponse aux critiques qui lui étaient faites dans le London Daily News du 20 janvier 1848, ainsi qu'un imprimé de 20 pages, Oxford Tradesmen Versus the Insolvent Jennings (1848), expliquant de façon circonstanciée l'affaire Jennings. Selon lui, la

27. Helen Berry, «Polite Consumption in Eighteenth-Century England», Transactions of the Royal Historical Society, t. 12, 2002, p. 377.

28. Rudi Laermans, "Learning to Consume : Early Department Stores and the Shaping of Modern Consumer Culture (1860-1914)», Theory, Culture \& Society, t. 10, n 4, 1993, p. 85.

29. Thomas Hughes, Tom Brown at Oxford, New York, MacMillan, [1861] 1888, p. 27. Drysdale est prêt à acheter des vêtements à un marchand venu directement dans sa chambre, mais ses amis lui font entendre raison. 
presse avait déformé les faits : il n'avait pas, comme l'affirmaient les journalistes, vendu dix chapeaux au jeune homme en un an puisque la somme qui devait lui être payée - relative uniquement aux chapeaux - s'élevait à $8,15 £$ s. sur un total de $70 £ 1$ s. 6 p. dus aux chapeliers. Randall dénonça le comportement irresponsable de Jennings, arguant que de nombreux marchands avaient tenté d'enrayer sa folie : selon lui, deux marchands de vin avaient refusé de lui fournir un nombre élevé de bouteilles de champagne et de clairette avant qu'il ne quitte la ville; d'autres avaient été prévenus que ses dépenses devenaient incontrôlables ${ }^{30}$. Si les marchands incitaient les étudiants à passer commande, il arrivait que leurs clients devinssent d'insatiables consommateurs ne pouvant s'abstenir d'acheter de nouveaux produits ou de s'offrir les services d'un prestataire ${ }^{31}$.

Le scandale Jennings, par son ampleur, ternit la réputation des marchands d'Oxford ${ }^{32}$ qui durent dès lors se défendre en publiant à leur tour des articles dans la presse locale. D'après leurs estimations, seul un pourcentage infime d'étudiants s'était endetté. Les archives de la cour de justice de l'Université prouvent qu'ils n'avaient pas entièrement tort : certes, environ $12 \%$ de la population estudiantine à Oxford dans les années $1840^{33}$ fut poursuivie en justice pour dette; cependant ce pourcentage ne veut pas dire pour autant que d'autres étudiants n'avaient pas également des dettes. En effet, ces dernières pouvaient très bien être remboursées par de généreux parents avant qu'ils n'obtinssent leur diplôme. Ainsi un certain nombre d'étudiants échappent aux statistiques. Soixante-dix marchands, ayant pour la grande majorité d'entre eux un magasin en centre-ville, donc près des collèges, se regroupèrent pour former une association et lutter contre les accusations de malhonnêteté dont ils étaient victimes. Selon le Kendal Mercury du 26 mai 1849, la plupart des marchands les plus opulents et bien établis à Oxford ne prirent pas la peine de participer à cette association. Ils refusèrent notamment de devoir écrire une lettre aux parents, prétextant que cette forme de délation serait préjudiciable à leur commerce. En effet, ils considéraient que les étudiants incriminés se sentiraient trahis et offensés, et que par conséquent ils inciteraient leurs amis à boycotter leur magasin. La proportion d'étudiants payant régulièrement leurs achats étant plus importante que celle cumulant des dettes, ils craignaient l'effet dévastateur que pouvait avoir une publicité négative à leur encontre, par le bouche-à-oreille notamment. La

30. Thomas Randall, Oxford Tradesmen Versus the Insolvent Jennings: A Verbatim Copy of the Schedule, Oxford, F. Trash, [1848], p. 25.

31. En effet, les factures conservées dans les archives de l'Université d'Oxford montrent que les étudiants pouvaient avoir recours à un barbier pour les raser ou pouvaient louer des couverts aux traiteurs lorsqu'ils commandaient de la nourriture pour un festin.

32. Certaines affaires avaient déjà fait les gros titres en 1821, notamment Fletcher vs. Sandiland (Morning Chronicle, 3 décembre 1821).

33. Statistiques réalisées dans le cadre de mon étude Young Men at Oxford. Routes Into Consumption and Debt, 1830-80, doctorat d'Histoire économique et sociale soutenu à l'Université d'Oxford en 2017. 
relation entre clients et marchands se distinguait donc par sa complexité, chacun pouvant faire pression sur l'autre.

Le 6 mai 1848 l'association informa les lecteurs de l'Oxford Journal qu' elle désirait réguler les dépenses des étudiants afin de pouvoir mieux les protéger contre des pratiques commerciales «sauvages». Un document d'archives datant du 27 mars 1848, rédigé par J. Vincent, libraire et membre de l'association, montre que certains marchands reconnaissaient que l'inexpérience des étudiants pouvait les conduire à être escroqués. De plus, selon eux, les pratiques commerciales qui consistaient à venir solliciter directement les jeunes hommes les poussaient par conséquent à acheter à crédit des biens de consommation qu'ils ne désiraient pas forcément. Après avoir discuté avec les tuteurs des principaux collèges, ils proposèrent que soient appliquées de nouvelles règles, qu'ils rendirent publiques en les publiant dans l'Oxford Journal du 19 mai 1848 : une notice devait tout d'abord être placardée à la porte de chaque collège et hall priant les jeunes consommateurs d'obtenir les factures de leurs fournisseurs deux fois par an; les membres de l'association devaient quant à eux s'abstenir de venir personnellement les importuner dans leur chambre; enfin ils exigeaient que la liste des étudiants en dernière année leur soit confiée afin de pouvoir relancer voire poursuivre en justice plus facilement les mauvais payeurs. Ces règles fixées par les marchands dans un effort de concertation avec l'Université d'Oxford, se voulaient préventives et tendaient à apaiser et normaliser les relations avec leurs clients. De fait, les factures devaient être envoyées régulièrement afin d'assurer une certaine transparence. En contrepartie, les marchands se réservaient le droit d'obtenir des informations confidentielles sur leurs débiteurs, qu'ils ne pouvaient avoir généralement qu'en donnant des pots de vins aux scouts des collèges (les serviteurs). Cependant, le Vice-Chancelier de l'Université refusa catégoriquement de répondre à cette dernière requête. La décision du Dr Wynter était d'importance : elle rendait vaines les attentes et les espérances des marchands. En effet, les étudiants pouvaient, tout comme l'avait fait Edward Jennings, décamper et se dérober à leurs responsabilités. De plus, s'enfuir en étant toujours mineur présentait certains avantages : les étudiants pouvaient plaider "l'immaturité" (infancy) devant la cour de justice de Londres et ainsi mieux échapper à leurs créanciers, leurs dettes étant le plus souvent annulées ${ }^{34}$.

D'après le Cambridge Independent (19 mai 1849), les marchands, frustrés de ne pouvoir obtenir satisfaction, menacèrent de se rendre à la Divinity School au moment de la remise des diplômes, remettant au goût du jour une ancienne tradition qui consistait à mettre son veto en tirant la toge du représentant du conseil de discipline de l'Université (proctor) lorsque le nom d'un

34. En effet, la loi interdisait aux marchands de vendre des produits de luxe aux mineurs. Ces règles avaient été également imposées par les autorités de l'Université d'Oxford. Voir : The Oxford University Statutes, Londres, Pickering, 1845. 
étudiant endetté était prononcé35. En effet, un étudiant ne pouvait obtenir sa licence s'il était redevable d'une somme d'argent. Ces menaces n'eurent que peu d'effet sur le Vice-Chancelier qui maintint sa décision et campa sur ses positions. L'association fut dissoute un an plus tard. Ainsi, malgré leurs efforts pour réguler le marché et imposer de nouvelles règles commerciales, certains marchands durent faire également face à une politique de laissezfaire instaurée par l'Université qui ne souhaitait pas se mêler des relations entre commerçants et étudiants. La concurrence demeura forte, le nombre de marchands qui s'installaient à Oxford augmentant inexorablement ${ }^{36}$, tandis que la pratique du démarchage tendit à diminuer dans les années qui suivirent l'affaire Jennings, les parents ayant été alertés par la presse et par les collèges ${ }^{37}$. L'utilisation du crédit à long terme s'avéra finalement l'un des moyens les plus commodes pour pousser les étudiants à (sur)consommer.

\section{Facilités de paiement et endettement}

Le crédit à la consommation joue un rôle majeur dans les sociétés modernes, permettant au consommateur d'acheter un bien sans pour autant posséder la somme totale devant être payée au moment de régler celuici. Comme le souligne Frank Trentmann dans son étude sur l'histoire de la consommation, les gens devinrent dépendants du crédit au cours du $\mathrm{xx}^{\mathrm{e}}$ siècle, sans avoir pourtant les moyens de payer les traites, et ce, afin de s'offrir des biens dont ils n'avaient pas véritablement besoin ${ }^{38}$. Lendol Calder va dans le même sens lorsqu'il évoque le développement du crédit aux ÉtatsUnis au début du $\mathrm{xx}^{\mathrm{e}}$ siècle. Selon lui, bénéficier d'un crédit signifiait que les consommateurs étaient tentés d'acheter des choses dont ils pouvaient se passer, et donc de s'endetter par "confort" (debt of convenience) $)^{39}$. Les pratiques commerciales semblent ainsi liées à l'idée de consumérisme - et non pas uniquement de consommation - de même qu'à l'endettement, celuici étant souvent le résultat de celui-là. Comment le crédit était-il utilisé à Oxford par les marchands et les étudiants? À quelles fins et quelles furent les conséquences de son utilisation? En quoi marque-t-il une transition vers une société de consommation et une adhésion au modèle capitaliste? 259.

35. Alan Crossley (dir.), Victoria History of the County of Oxford, Londres, 1979, vol. 4, p. 181-

36. 720 commerces en 1823 contre 2628 en 1891 (chiffres provenant des annuaires Pigot et Kelly). Voir la note 4.

37. En 1848, F. C. Plumptre, Master de University College, rédigea une lettre pré-imprimée qu’il fit envoyer aux parents (University College Archives, réf. MA40/N1/1).

38. Frank Trentmann, The Empire of Things. How We Became a World of Consumers from the Fifteenth to the Twenty-First, Allen Lane, Penguin books, 2016, p. 405.

39. Lendol Calder, Financing the American Dream: A Cultural History of Consumer Credit, Princeton, NJ, Princeton University Press, 1999, p. 103. 
Comme l'ont montré les travaux de Paul Johnson, le crédit permettait aux classes populaires et au prolétariat de faire face à des difficultés financières temporaires ${ }^{40}$. Emprunter de l'argent était perçu alors comme une faillite à la fois morale et sociale, et compris comme une incapacité à gérer son budget. En revanche, que l'aristocratie désirât acquérir des biens très onéreux en ayant recours au crédit semblait parfaitement naturel car vu comme un privilège de classe. Or cette forme de crédit de confort devint de plus en plus fréquente, et ce, dès le XviII ${ }^{\mathrm{e}}$ siècle comme le suggère Edward Mangin ${ }^{41}$. Elle semble avoir été un phénomène essentiellement urbain, les commerçants offrant à la bourgeoisie et à la noblesse des facilités de paiement ${ }^{42}$. «Le public, pouvant aller chez le boucher, le boulanger, le maraîcher, le tailleur etc. sans argent, et pouvant être vêtu et nourri par eux, est conduit à gaspiller de l'argent et dépenser de façon extravagante» asserta l'Essex Standard du 26 novembre $1847^{43}$. Le crédit offert de façon systématique avec en contrepartie le paiement d'intérêts entrait dans les mœurs. Les classes supérieures tout comme la classe moyenne trouvaient un avantage indéniable à régler leurs factures par échéance et de façon groupée. Les étudiants, dont la grande majorité provenait de milieux aisés, utilisèrent le crédit à long terme qui leur était offert par les marchands, à la fois comme une commodité et comme un moyen efficace pour juguler leurs dettes.

Le débat sur le crédit à la consommation ne fut ouvert qu'à partir des années 1840, au moment même où les premiers procès d'étudiants ruinés faisaient les gros titres des journaux. En effet, les marchands d'Oxford ayant une clientèle particulière, jeune et le plus souvent riche, systématisèrent le crédit, quand bien même ces jeunes hommes n'auraient pas de revenus et resteraient très dépendants de leurs parents. Les étudiants se voyaient offrir un crédit courant sur une, deux, voire plusieurs années, ce qui s'avérait des plus pratiques, d'autant que leur compte n'était pas plafonné. Dès leur arrivée à Oxford, ils pouvaient donc commander ce que bon leur semblait. Il suffisait de se présenter à la boutique ou de passer commande, souvent par l'intermédiaire du scout, depuis sa chambre en donnant son nom et son collège.

40. Paul Johnson, Saving and Spending: The Working-Class Economy in Britain, 1870-1939, Oxford, Oxford University Press, 1985. Voir également son article : "Small Debts and Economic Distress in England and Wales, 1857-1913", Economic History Review, t. 46, n 1, 1993, p. 69.

41. Edward Mangin, A Few General Directions for the Conduct of Young Gentlemen in the University of Oxford, Oxford, 1795, réed. Londres, James Carpenter, 1807, p. 73 ("At present in Oxford, money is nearly useless").

42. Cette forme de crédit de confort différait du crédit traditionnel propre à l'économie «morale» (voir Craig Muldrew, "Interpreting the Market : The Ethics of Credit and Community Relations in Early Modern England», Social History, t. 18, n 2, 1993, p. 163-183). En effet, ce type de crédit "moderne" était fondé sur un contrat entre créditeur et débiteur. Par ailleurs, le client devait payer en général $5 \%$ d'intérêts par an sur la somme restant à rembourser. L'ancien crédit était fondé sur un accord passé oralement entre deux personnes se faisant mutuellement confiance («l'ardoise»). Cet accord n'impliquait pas forcément le paiement d'intérêts à court ou moyen terme.

43. "The public, finding they can go to their butcher, baker, grocer, tailor, etc. without money, and by them can be clothed and fed, are led into wasteful expenditure and profligate extravagance». 
Les marchands se gardaient de s'enquérir trop ouvertement de leurs moyens financiers, gageant le plus vraisemblablement que les parents paieraient les dettes par crainte du déshonneur. De fait, beaucoup de jeunes hommes devenaient en quelque sorte consommateurs de crédit, vivant à crédit comme le déplora l'auteur de Tradesmen and Undergraduates lorsqu'il évoquait ceux qu'il nommait les hommes "empressés» de dépenser (fast men) :

"Ainsi se passent les deux premières années de l'homme empressé; ses dettes s'accumulant silencieusement de toutes parts et certaines d'entre elles atteignant déjà un montant conséquent. Bien qu'il soit de temps à autre alarmé par la perspective d'avoir de futurs ennuis, il ne se presse pas en général de payer; tout du moins jusqu'au moment où il doit passer son diplôme. Alors sa situation est des plus pitoyables. Factures, menaces d'arrestation, lettres des comptables le cernent de toutes parts. Des centaines de factures d'un montant dérisoire qu'il avait oubliées depuis longtemps ou même imaginé de s'être acquitté pleuvent chaque jour sur la table où il prend son petit-déjeuner. [...] Persécuté et dérangé alors qu'il devrait être nécessairement plongé dans ses études, il commet alors une erreur encore plus grave, et s'en remet aux usuriers, desquels il obtient à des taux d'intérêts exorbitants et souvent compromettant son avenir, la somme d'argent qui lui est nécessaire pour rembourser les créanciers qui le pressent le plus. Ce jour sordide n'est cependant que remis à plus tard. Tôt ou tard ses parents ont vent de ses ennuis financiers, et alors commence le bras de fer ${ }^{44}$ ».

Selon toute vraisemblance, l'endettement des étudiants les plus désinvoltes se produisait en trois étapes : l'ouverture d'un compte auprès d'un marchand permettait d'acheter à crédit, d'où le sentiment de pouvoir satisfaire tous ses désirs; la multiplication d'achats et donc l'accumulation de sommes dues à différents marchands pendant une longue période creusait de façon subreptice son endettement; et finalement le recours aux usuriers ${ }^{45}$ pour rembourser ses dettes menait au surendettement et à la ruine. Comme on peut le voir dans cet extrait, le crédit semble être à double-tranchant $: s^{\prime}$ il s'avère pratique, il peut être cependant source de graves problèmes lorsqu'il

44. "Thus pass the two first years of the 'fast' Oxford man, his debts silently accumulating on every side, and some of them already grown to a very serious amount. Although every now and then forewarned of his coming troubles by some wishes for upon the old terms, nor is he in general much pressed for payment until the time approached for him to take his degree. Then, indeed, his situation is truly pitiable. Bills, threats of arrest, accountants' letters, beset him on every side. Hundreds of little paltry claims which he has long since forgotten or imagined to have been paid, are showered daily upon his breakfast table [...]. Thus persecuted and disturbed in his necessary studies, he often falls into even a worse error, and betakes himself to the money lenders from whom, at enormous interest, and often to the serious detriment of his future prospects, he obtains wherewith to satisfy the most urgent of his creditors. The evil day is, however, but postponed. Sooner or later the news of his embarrassment reaches the ear of his father or guardian, and then comes the tug of war ». (Tradesmen and Undergraduates, op. cit., p. 7-9). Nous traduisons.

45. Il ne nous a pas été possible de déterminer le nombre d'usuriers à Oxford dans la mesure où la plupart restait dans l'ombre. Ils ne sont pas recensés dans les annuaires Pigot ou Kelly par exemple. 
est mal géré, que cela soit avec le même ou avec différents marchands. D'où l'empressement des créanciers à vouloir récupérer leur argent, et d'où leur harcèlement vis-à-vis des mauvais payeurs (duns) ou de ceux dont les commandes s'espaçaient dangereusement. En effet, ne plus donner signe de vie paraissait toujours de mauvais augure pour le commerçant : l'étudiant pouvait avoir trouvé un meilleur fournisseur et donc délaissé son magasin; il pouvait délibérément refuser de payer ses dettes en ignorant les factures qui lui étaient envoyées ou pire, être sur le point de quitter la ville. L'insistance avec laquelle les marchands pouvaient se mobiliser pour récupérer l'argent qui leur était dû entraînait souvent de nouvelles commandes; dans certains cas leurs démarches avaient précisément cette visée comme le prétendait James Pycroft ${ }^{46}$, les marchands menaçant de clore les comptes. Les étudiants ne pouvant payer immédiatement une somme conséquente préféraient acheter de nouveaux biens de consommation. C'est pourquoi l'Oxford University and City Herald du 9 juillet 1842 avait relevé les dangers liés au crédit, le considérant comme "une machine à extorquer et à chicaner».

En séparant l'achat d'un bien et son paiement, les marchands d'Oxford avaient mis en place un système visant à stimuler la demande de façon artificielle et donc à pousser à la consommation. Comme le rappelait l'Oxford Chronicle and Reading Gazette du 20 octobre 1849 - soit un an après l'affaire Jennings -, l'offre devait toujours répondre à la demande. Le crédit à long terme, parce qu'étant une pratique commerciale dominante à Oxford, avait un rôle majeur dans le développement économique. Le système pouvait d'ailleurs s'étendre et s'accroître dans la mesure où le nombre d'étudiants, de même que celui des habitants et des commerçants, ne cessa d'augmenter au $\mathrm{XIX}^{\mathrm{e}}$ siècle ${ }^{47}$. Une culture consumériste put ainsi voir le jour dès la première moitié du siècle, pour s'enraciner par la suite, le niveau de vie s'améliorant d'ailleurs progressivement à l'échelle nationale et le désir de vivre confortablement se traduisant par un intérêt croissant pour les biens matériels ${ }^{48}$. Les étudiants pouvaient aussi, en achetant à crédit, accumuler ceux-ci pour ensuite les revendre, un marché parallèle existant probablement à Oxford ${ }^{49}$.

46. Nous traduisons : "three or four times in about as many weeks I was pestered for orders by a man who brought round fancy patterns for trousers and waistcoats. I was continually importuned not to pay my old debt, but to contract new ". (James Pycroft, Oxford Memories, A Retrospect after Fifty Years, London, Richard Bentley \& Son., 1886, p. 246-247). James Pycroft étudia à Trinity College en 1831 et obtint sa licence en 1836 .

47. $1811: 13254 ; 1831: 20710 ; 1851: 27843$ habitants et $1871: 31404$ habitants (noter que lors du recensement en 1871 , les membres de l'université étaient en vacances et que le nombre d'habitants est par conséquent légèrement sous-estimé). Ces données sont tirées de A. Crossley (dir.), Victoria History of the County of Oxford, Londres, 1979 et sont consultables en ligne à l'adresse suivante : http://www.british-history.ac.uk/vch/oxon/vol4/pp181-259.

48. Comme le soulignent Sabine Efosse et Isabelle Gaillard, "le crédit à la consommation est indissociable de l'avènement des biens de consommation durables ou semi-durables dans l'entredeux-guerres, de la hausse du niveau de vie et de la société de loisirs et de services qui caractérise la fin de siècle» : "L'Europe et le crédit à la consommation", Entreprises et histoire, t. 56, n 2, 2010, p. 5.

49. Les étudiants pouvaient également payer en argent liquide mais la plupart des marchands, 
De fait, le marché se caractérisait par une certaine volatilité qui peut expliquer la nervosité des marchands et leur volonté de «sécuriser " à la fois de nouvelles commandes et leur paiement. Le crédit pouvait également être risqué pour eux : ils devaient veiller à ne pas avoir de dettes trop élevées auprès de leurs fournisseurs. De plus, étant un moteur micro-économique, le système du crédit instauré par les marchands préfigurait des formes sophistiquées de capitalisme. La transition vers des paiements en argent comptant fut lente à Oxford, quand bien même l'essor du grand magasin à la fin de l'époque victorienne aurait permis une évolution des modes de paiement ${ }^{50}$. Néanmoins, cette offre et utilisation systématique du crédit permet de mieux comprendre comment a pu se mettre en place la consommation de masse : un tel mécanisme, reproduit à une plus grande échelle, pouvait amener les clients à développer des habitudes consuméristes et à acheter de façon continue des biens alors qu'ils n'en avaient pas forcément les moyens. Or c'est bien ce phénomène qui apparaît au début du $\mathrm{Xx}^{\mathrm{e}}$ siècle, comme l'a montré Calder, et qui financera notamment les rêves matérialistes des consommateurs américains.

$*$

En conclusion, les années 1840 furent marquées par une période d'intense concurrence entre marchands à Oxford et par des pratiques commerciales agressives qui visaient spécifiquement les étudiants, mais qui témoignaient également du manque de régulation et de réglementation du marché. Si l'affaire Jennings ne fut pas exceptionnelle, elle fut néanmoins un tournant dans l'histoire d'Oxford, en ce qu'elle permit de mettre à jour certaines pratiques douteuses et de révéler au public l'endettement de certains étudiants. L'Université d'Oxford refusa de servir de médiateur entre étudiants et marchands, opposant une fin de non-recevoir aux demandes qui avaient été formulées, alors que ces derniers tentaient de coopérer avec les collèges et d'établir de nouvelles règles commerciales plus transparentes afin de sauver leur réputation. La presse, de façon systématique, dénigra les commerçants. L'insolvabilité de certains jeunes hommes devint un sujet de préoccupation au niveau national, aussi le député Thomas Phinn ${ }^{51}$ proposa par exemple en 1854 de restreindre les dépenses des étudiants mineurs en interdisant aux marchands

comme en témoignent les factures présentes dans les dossiers de la Chancellor's Court à Oxford, préféraient faire crédit à leur clientèle.

50. C'est ce que soutient Margot Finn : The Character of Credit. Personal Debt in English Culture, 1740-1914, Cambridge, Cambridge University Press, 2003, p. 278. Certains magasins comme Elliston and Cavell ou Caper voulurent imposer des transactions en argent comptant mais ces tentatives s'avérèrent infructueuses.

51. Thomas Phinn (1814-1866) était un ancien étudiant d'Eton et d'Exeter Collège. Il devint avocat, puis fit de la politique pour le Liberal Party et devint député en 1852. Voir Oxford University Bill, House of Commons, Deb. 22 June 1854, vol. 134 cc511-95, consultable en ligne : http:// hansard.millbanksystems.com/commons/1854/jun/22/oxford-university-bill\#column_512. 
de les poursuivre en justice. Sa proposition fut rejetée car il existait déjà une loi votée en 1849 frappant de nullité tout achat effectué à crédit par un mineur - loi qui ne paraît pas cependant avoir été appliquée à Oxford, la Chancellor's Court ayant ses propres lois et fonctionnement. Le crédit fut aussi remis en question. Avoir recours à celui-ci ne signifiait pas que les étudiants étaient sans ressources ou à court d'argent, mais qu'ils tiraient parti des facilités de paiement qui leur étaient proposées. Ils achetaient donc quand bon leur semblait et ce qui leur faisait envie quel qu'en soit le prix. Le crédit à long terme fut ainsi le plus souvent, pour un certain nombre d'étudiants, à l'origine de graves difficultés financières, d'autant que les marchands offraient un crédit illimité 52 . Comme Clive Edwards le montre, avoir accès au crédit à la consommation permit l'avènement d'une société moderne et consumériste ${ }^{53}$. Contrairement à ce qu'affirment Rosa-Maria Gelpi, François Juline-Labruyère et Liam Gavin, le crédit impacta le niveau de consommation, plutôt que la consommation n'agit sur le développement du crédit. Les étudiants ne devenaient pas seulement des consommateurs mais également des consommateurs de crédit, celui-ci ayant été "standardisé» par les marchands d'Oxford et étant devenu un "contrat» (plutôt qu'un accord passé oralement avec le client comme par le passé). Le crédit était ainsi enraciné dans la vie estudiantine et dans le quotidien des habitants de la ville. Un étudiant remarqua d'ailleurs : «je dois [était] trop fréquemment entendu dans la bouche des jeunes hommes venus à Oxford " $\$ 4$.

Il semble que les étudiants trouvèrent un moyen judicieux d'échapper à l'emprise du crédit et à la spirale de dettes dans laquelle beaucoup d'entre eux étaient entraînés. En effet, à la veille de la Première Guerre mondiale, le Manchester Evening informa ses lecteurs de l'ouverture prochaine d'une coopérative estudiantine :

«Les étudiants d'Oxford s'associent aux professeurs des collèges afin de mettre à bas le système du crédit à l'Université et le remplacer par une entreprise dirigée par les étudiants et fonctionnant sur un mode coopératif. Un magasin doit être ouvert au prochain trimestre, au n ${ }^{\circ} 20$ sur High Street. Il sera approvisionné en papèterie, articles de sport, produits alimentaires et accessoires pour les fumeurs. Des membres plus âgés de l'Université, ainsi que des étudiants en licence auront des parts dans cette société nouvelle et tous les profits réalisés seront partagés et redistribués entre les actionnaires, proportionnellement à leur investissement. La société sera affiliée à la coopérative principale et à la Industrial Society, avec laquelle a été passé un accord concernant les achats ${ }^{55}$."

52. The Oxford Undergraduate's Journal, 10 novembre 1866.

53. Clive Edwards, "Buy Now - Pay later. Credit: The Mainstay of the Retail Furniture Business?", in John Benson et Laura Ugolini (dir), Cultures of Selling : Perspectives on Consumption and Society since 1700, Aldershot, Ashgate, 2006, p. 128.

54. The Oxford Undergraduate's Journal, 10 novembre 1866.

55. «Oxford Undergraduates are joining forces with college dons to put down the credit 
Cette volonté de contrôler leur propre consommation et leurs dépenses en devenant eux-mêmes des commerçants suggère que les étudiants ne voulaient plus désormais être "traités comme des pigeons"; au contraire, ils aspiraient à un commerce plus équitable et à un marché mieux équilibré où ils auraient un rôle actif au niveau de la demande et de l'offre, et qui leur permettrait finalement de devenir plus indépendants et plus responsables.

\section{Sabine Chaouche est professeure d'histoire culturelle et Doyenne associée, Sunway University, Malaisie.}

ANNEXE

Affaire Jennings

Liste des créanciers et liste des factures ${ }^{56}$

\begin{tabular}{|l|l|l|l|l|l|l|}
\hline \multicolumn{1}{|c|}{ Année } & $\begin{array}{c}|c| \\
N^{\circ} \text { du } \\
\text { marchand }\end{array}$ & \multicolumn{1}{|c|}{ Profession } & \multicolumn{3}{|c|}{ Somme due* } \\
\hline & & & & f & S & d \\
\hline 1846 & 7 & Thomas Betteris & Billards & 0 & 15 & 0 \\
\hline 1847 & 74 & James Leaver & Palfrenier & 0 & 16 & 0 \\
\hline 1846 & 77 & F. Boswell & Chapelier & 0 & 17 & 0 \\
\hline 1846 & 3 & George Bridges & Vendeur de jouets & 1 & 13 & 6 \\
\hline 1847 & 9 & W. Brown & Pâtissier & 1 & 17 & 4 \\
\hline 1847 & 12 & Castle & Marchand de tabac & 1 & 10 & 0 \\
\hline $1845-6-7$ & 59 & William Smith & Propriétaire d'écurie & 1 & 8 & 0 \\
\hline 1846 & 5 & S. Beesley & Propriétaire d'écurie & 2 & 8 & 0 \\
\hline $1846-7$ & 14 & Coles & Coiffeur & 2 & 0 & 0 \\
\hline 1847 & 22 & T. Greatbach & Propriétaire d'écurie & 2 & 10 & 0 \\
\hline 1846 & 34 & Samuel Hester & Valet d'écurie & 2 & 2 & 0 \\
\hline $1845-6-7$ & 36 & William Luker & Vendeur d'animaux & 2 & 9 & 0 \\
\hline $1845-7$ & 41 & John Henry Parker & Libraire & 2 & 9 & 6 \\
\hline $1845-6$ & 46 & Rose & Bonnetier & 2 & 0 & 0 \\
\hline $1846-7$ & 65 & Shillingford \& Phillips & Marchand de bière & 2 & 5 & 0 \\
\hline $1845-6$ & 71 & Williams & Tailleur & 2 & 5 & 6 \\
\hline & & & & & \\
\hline
\end{tabular}

system in the university to establish in its stead a business conducted mainly by undergraduates on co-operative lines. A shop is to be opened next term at 20, High Street and will be stocked with stationery, athletic goods, groceries, and smokers' requisites. The shares in the new society will be held by senior and undergraduate members of the university and those undergoing and all profits will be distributed among the shareholders in proportion to the amount of their purchases. The society will be affiliated to the chief co-operative and Industrial Society, with which it has entered into mutual purchasing arrangement». Nous traduisons.

56. Tirée de Oxford Tradesmen Versus the Insolvent Jennings, op. cit. 


\begin{tabular}{|c|c|c|c|c|c|c|}
\hline Année & $\begin{array}{c}\mathrm{N}^{\circ} \mathrm{du} \\
\text { marchand }\end{array}$ & Nom & Profession & \multicolumn{3}{|c|}{ Somme due* } \\
\hline 1847 & 75 & Arthur Smith & Vendeur d'animaux & 2 & 2 & 0 \\
\hline $1845-6-7$ & 23 & Levi Greatbach & Vendeur de porcelaines & 3 & 7 & 6 \\
\hline $1846-7$ & 30 & E. Hickman & Opticien & 3 & 3 & 0 \\
\hline 1846-7 & 33 & Hackshaw & Coiffeur & 3 & 0 & 0 \\
\hline $1845-6-7$ & 62 & Carter, Sherratt \& Hall & Loueur de bateaux & 3 & 0 & 0 \\
\hline 1845-6-7 & 64 & Samuel Steane & Marchand de bière & 3 & 4 & 6 \\
\hline $1845-6-7$ & 66 & Sheard & Vendeur de thé & 3 & 0 & 0 \\
\hline 1847 & 70 & Lawrence Wyatt & Vendeur d'animaux & 3 & 4 & 0 \\
\hline $1846-7$ & 4 & Bickerton & Billards & 4 & 0 & 0 \\
\hline $1845-6$ & 44 & Plowman & Quincailler & 4 & 0 & 0 \\
\hline $1845-6-7$ & 52 & S. Sutton & Vendeur de porcelaines & 4 & 1 & 0 \\
\hline 1847 & 6 & W. Blackwell & Sellier & 5 & 3 & 6 \\
\hline $1845-6$ & 16 & Charles Davis & Propriétaire d'écurie & 5 & 3 & 0 \\
\hline $1845-6$ & 25 & John Gillam & Hôtelier & 5 & 2 & 6 \\
\hline $1845-6-7$ & 63 & Shrimpton & Imprimeur & 5 & 0 & 0 \\
\hline 1846-7 & 79 & Young & Quincailler & 5 & 0 & 0 \\
\hline 1845 & 28 & Hounslow & Épicier & 6 & 7 & 2 \\
\hline 1847 & 37 & Latimer & Marchand de vin & 6 & 0 & 0 \\
\hline $1845-6$ & 45 & Quaterman & Fabricant de culottes & 6 & 0 & 0 \\
\hline 1846 & 42 & Joseph Prior & Tailleur & 7 & 12 & 6 \\
\hline $1846-7$ & 69 & Vaughan & Boulanger & 7 & 18 & 8 \\
\hline $1845-6-7$ & 18 & Dewe & Bibliothécaire & 8 & 0 & 0 \\
\hline $1845-6$ & 24 & William Graham & Libraire & 8 & 3 & 0 \\
\hline 1845 & 55 & E. Standen & Fabricant de chemises & 8 & 17 & 3 \\
\hline 1847 & 60 & Joseph Sabin & Libraire & 9 & 9 & 6 \\
\hline $1845-6-7$ & 39 & Oxford Wine Company & Marchand de vin & 10 & 5 & 6 \\
\hline $1846-7$ & 76 & Beckuson & Fabricant d'armes & 10 & 14 & 3 \\
\hline $1845-6-7$ & 54 & K.S. Standen & Fabricant de chemises & 11 & 15 & 6 \\
\hline $1845-6-7$ & 68 & H.S. Underhill & Épicier & 11 & 4 & 2 \\
\hline $1846-7$ & 10 & Bryant & Marchand de tabac & 13 & 4 & 1 \\
\hline $1845-6-7$ & 50 & Henry Slatter & Libraire & 13 & 15 & 2 \\
\hline $1845-6-7$ & 32 & $\begin{array}{l}\text { William Herbert \& Richard } \\
\text { Embling }\end{array}$ & Tapissiers & 15 & 2 & 6 \\
\hline $1846-7$ & 17 & J.W. Dickeson & Maison de thé & 16 & 10 & 10 \\
\hline
\end{tabular}




\begin{tabular}{|l|l|l|l|l|l|l|}
\hline \multicolumn{1}{|c|}{ Année } & $\begin{array}{c}N^{\circ} \text { du } \\
\text { marchand }\end{array}$ & \multicolumn{1}{|c|}{ Nom Profession } & \multicolumn{3}{c|}{ Somme due* } \\
\hline $1846-7$ & 21 & J. Fiske \& Son & Tailleurs & 16 & 7 & 0 \\
\hline 1847 & 8 & J. Bennett \& Co & Tailleur & 17 & 10 & 0 \\
\hline $1845-6$ & 40 & Perkins & Marchand de vin & 17 & 11 & 6 \\
\hline $1845-6-7$ & 49 & Thomas Slatter & Tailleur & 20 & 13 & 6 \\
\hline $1845-6-7$ & 57 & John Simms & Fabricant de chaussures & 22 & 8 & 6 \\
\hline $1846-7$ & 56 & William Slark & Sellier & 23 & 0 & 9 \\
\hline $1845-6-7$ & 11 & Nathaniel Castle & Chapelier & 27 & 3 & 0 \\
\hline $1845-7$ & 58 & Seckham & Propriétaire d'écurie & 27 & 0 & 0 \\
\hline $1846-7$ & 29 & John Harwood & Fabricant de chaussures & 28 & 17 & 6 \\
\hline $1845-6-7$ & 61 & Spiers \& Son & Papetier et dépôt & 30 & 16 & 9 \\
\hline $1846-7$ & 53 & P. Slatter & Hôtelier & 31 & 10 & 0 \\
\hline $1845-6-7$ & 15 & T.G. Cousins & Apothicaire & 32 & 10 & 8 \\
\hline $1846-7$ & 20 & James Embling & Tailleur & 34 & 6 & 6 \\
\hline $1845-6-7$ & 72 & Robert Wharton & Pâtissier & 41 & 14 & 4 \\
\hline $1845-6-7$ & 38 & T.W. Martin & Tailleur & 44 & 15 & 6 \\
\hline $1845-6-7$ & 67 & W. Thompson & Imprimeur & 46 & 4 & 3 \\
\hline $1845-6-7$ & 47 & Randall & Chapelier & 50 & 0 & 0 \\
\hline $1845-6-7$ & 26 & Hookham \& Minty & Tailleur & 82 & 8 & 0 \\
\hline $1845-6-7$ & 31 & Hobdell & Bijoutier & 97 & 0 & 0 \\
\hline $1845-6-7$ & 51 & G. Symonds & 146 & 0 & 0 \\
\hline
\end{tabular}

* Livre, shilling, penny 
